Article

\title{
Gut Bacterial and Fungal Communities of the Wild and Laboratory-Reared Thitarodes Larvae, Host of the Chinese Medicinal Fungus Ophiocordyceps sinensis on Tibetan Plateau
}

\author{
Guiqing Liu (®, Xuehong Zheng, Hailin Long, Zhongchen Rao, Li Cao and Richou Han * \\ Guangdong Key Laboratory of Animal Conservation and Resource Utilization, \\ Guangdong Public Laboratory of Wild Animal Conservation and Utilization, Institute of Zoology, Guangdong \\ Academy of Sciences, Guangzhou 510260, China; liugq@giz.gd.cn (G.L.); zxh1234560716@163.com (X.Z.); \\ hlin_long@163.com (H.L.); xipredus@163.com (Z.R.); caol@giz.gd.cn (L.C.) \\ * Correspondence: hanrc@giz.gd.cn
}

check for updates

Citation: Liu, G.; Zheng, X.; Long, H.; Rao, Z.; Cao, L.; Han, R. Gut Bacterial and Fungal Communities of the Wild and Laboratory-Reared Thitarodes Larvae, Host of the Chinese Medicinal Fungus Ophiocordyceps sinensis on Tibetan Plateau. Insects 2021, 12, 327. https://doi.org/10.3390/ insects12040327

Academic Editor: Guy Smagghe

Received: 25 February 2021

Accepted: 29 March 2021

Published: 7 April 2021

Publisher's Note: MDPI stays neutral with regard to jurisdictional claims in published maps and institutional affiliations.

Copyright: (c) 2021 by the authors. Licensee MDPI, Basel, Switzerland. This article is an open access article distributed under the terms and conditions of the Creative Commons Attribution (CC BY) license (https:/ / creativecommons.org/licenses/by/ $4.0 /)$.
Simple Summary: The ghost moth, Thitarodes sp., is an obligate host of the most precious fungus Ophiocordyceps sinensis on Tibetan plateau. Artificial rearing of the ghost moth at low-altitude laboratory by mimicking the environment of the wild habitat for the cultivation of the Chinese cordyceps has been realized. However, the high mortality of ghost moth larvae by pathogens, low and slow infection, and mummification rate by $O$. sinensis still constrain the efficient cultivation of the Chinese cordyceps. Both larval gut microbiota and their exploitation in the Thitarodes artificial rearing system have attracted a renewed interest. In the present study, the gut bacterial and fungal communities of the wild and laboratory-reared populations were characterized using both culturedependent and -independent approaches. The discovery of apparent microbial community shifts between the wild and laboratory-reared ghost moth larvae, many opportunistic pathogenic bacteria and fungi in the gut of the laboratory-reared ghost moth larvae, and the dominant bacteria enriched in the wild ghost moth provide interesting cues for selecting beneficial probiotic bacteria to improve the effectiveness of Thitarodes rearing system and the cultivation of the Chinese cordyceps.

Abstract: By employing a culture-dependent and -independent 16S rRNA and ITS gene highthroughput sequencing analyses, comprehensive information was obtained on the gut bacterial and fungal communities in the ghost moth larvae of three different geographic locations from highaltitude on Tibet plateau and from low-altitude laboratory. Twenty-six culturable bacterial species belonging to 21 genera and 14 fungal species belonging to 12 genera were identified from six populations by culture-dependent method. Carnobacterium maltaromaticum was the most abundant bacterial species from both the wild and laboratory-reared larvae. The most abundant OTUs in the wild ghost moth populations were Carnobacteriaceae, Enterobacteriaceae for bacteria, and Ascomycota and Basidiomycota for fungi. Larval microbial communities of the wild ghost moth from different geographic locations were not significantly different from each other but significant difference in larval microbial community was detected between the wild and laboratory-reared ghost moth. The larval gut of the wild ghost moth was dominated by the culturable Carnobacterium. However, that of the laboratory-reared ghost moth exhibited significantly abundant Wolbachia, Rhizobium, Serratia, Pseudomonas, and Flavobacterium. Furthermore, the larval gut of the wild ghost moth had a significantly higher abundance of Ophiocordyceps but lower abundance of Candida and Aspergillus than that of the laboratory-reared ghost moth.

Keywords: ghost moth; Thitarodes; Ophiocordyceps sinensis; microbiota; high-altitude; artificial rearing

\section{Introduction}

Thitarodes/Hepialus ghost moths (Lepidoptera: Hepialidae) are obligate hosts of the medicinal fungus Ophiocordyceps sinensis, and the fungus-insect parasitic complex named 
Chinese cordyceps has been one of the most valued health foods and traditional Asian medicines since the 15th century ([1-3]. Ghost moths belonging to the primitive lepidopteran lineage [4] are endemic to the Tibetan plateau in alpine and subalpine regions at altitudes from 3000-5200 m with a specific high-altitude environmental condition characterized by hypoxia, low air pressure, low temperature, and high ultraviolet radiation intensity [5-7]. The ghost moth larvae mainly inhabit subterranean tunnels built by themselves around the plant roots for more than 3 years [8-10]. The larvae feed mainly on plant roots underground, maintain the feeding activity at $0{ }^{\circ} \mathrm{C}$ or a few degrees above zero, and encounter various pathogens such as fungi, bacteria, nematodes, predatory mites, and some other small insects [11-13].

The underlying mechanism of the adaptation to the high-altitude environment of the ghost moth, and their coexistence with the fungus $O$. sinensis has attracted much attention in recent years. Transcriptome analyses using high throughput sequencing have provided in-depth insights into the mechanism of environmental adaptation. In response to cold, metabolic rate and respiratory quotient decrease [14]. Differentially expressed genes associated with altitude were annotated in the process of lipid metabolism, carbohydrate metabolism, and respiration [15]. The ghost moth T. armoricanus might adopt a strategy to adapt to hypoxia by suppressing hypoxanthine catabolism, TCA, and oxidative phosphorylation pathways [16]. In response to O. sinensis infection, only a proportion of infected larvae could turn into stiff worms and become Chinese cordyceps even though the larvae were sampled from the same site or reared at the same conditions $[17,18]$. It appeared that the immune system and gut microbiota are involved in the pathogenicity of O. sinensis fungus [19-21].

Insect gut is the house of diverse microbiomes, which are evolving to play important roles in insect physiology and ecology, supporting growth, development, and survival of their hosts [22-24]. In general, gut microbiota can contribute to the nutrition provision for host [25], host immunity [26], host behavior [27], and mediate detoxification of plant secondary compounds [28,29] and insecticides [30]. Conversely, multiple factors such as host, environment, and diet can shape the structure of gut microbial communities [31-35]. Nevertheless, all these studies are based on the gut microbiota of low-altitude insect hosts.

So far, artificial rearing of the ghost moth at low-altitude laboratory by mimicking environment conditions of the wild habitat for the cultivation of the Chinese cordyceps at commercial scale has been successfully established $[3,36]$. However, the high mortality of ghost moth larvae by pathogens, low and slow infection, and mummification rate by O. sinensis still constrain the efficient production of the Chinese cordyceps [2,37,38]. Given the important role of the gut microbes, researchers attempt to identify microbes from the gut of the ghost moth larvae, hoping to find beneficial microbes for the artificial rearing ghost moth larvae and promoting the infection and mummification rate by $O$. sinensis. However, only 8 bacterial and 3 fungal genera were isolated from the wild T. gonggaensis larvae $[39,40]$ and 11 bacterial and 3 fungal genera were isolated from the laboratory-reared T. xiaojinensis [21] by the culture-dependent method. Furthermore, only Carnobacterium and Pseudomonas were common in the wild T. gonggaensis larvae and the laboratory-reared T. xiaojinensis. Therefore, it is necessary to comprehensively compare the gut microbiota between the wild and laboratory-reared ghost moth.

To explore the involvement of the gut microbiota in host physiology and pathogenicity, the larval gut bacterial and fungal communities of the wild ghost moth collected at alpine meadow on Tibet plateau and laboratory-reared ghost moth among three different geographic locations were characterized using both culture-dependent and cultureindependent approaches.

\section{Materials and Methods}

\subsection{Ghost Moth Collection, Identification and Gut Preparation}

The wild ghost moth 5th instar larvae (average fresh weight $=0.49 \pm 0.08 \mathrm{~g}$ ) were sampled from the known distribution areas of the Chinese cordyceps. Samples were 
collected from soil beneath alpine meadow from three different geographic locations during the harvesting seasons (May-July 2019) at altitudes $3500 \mathrm{~m}$ above sea level on the Tibetan plateau in Sichuan Province, China (See Figure S1 for details). The larvae from the above three different locations were named W.SD, W.GG and W.XJ populations, respectively according to their geographic location (Figure S1). The alive larvae were collected and placed individually in the vial filled with moss and sent to the laboratory in foam boxes with ice packs to maintain the low temperature.

The laboratory-reared ghost moth larvae were derived from the pupae collected from the above three geographic locations and reared at $9 \sim 13^{\circ} \mathrm{C}$ in Guangzhou, China according to the described methods [21,41]. The hatched larvae were offered the roots of Potentilla anserine as food to obtain 5 th instar larvae (average fresh weight $=0.57 \pm 0.07 \mathrm{~g}$ ) for gut microbial isolation. The laboratory-reared larvae from the above three different locations were named as A.SD, A.GG, and A.XJ populations, correspondingly.

To identify the ghost moths, the genomic DNA of the wild and laboratory-reared ghost moths were extracted using TaKaRa MiniBEST Universal Genomic DNA Extraction Kit (Takara Bio Inc, Dalian, China) and used as a template to amplify the mitochondrial cytochrome b gene (cytb) with the primers CB1 (TATGTACTACCATGAGGACAAATATC) and CB2 (ATTACACCTCCTAATTTATTAGGAAT) [42]. Then, the phylogenetic tree of the ghost moths from three different locations was constructed using neighbor-joining method in the MEGA 5.1 with a bootstrap value of 1000 .

All the larvae were dissected within 3 days after collection. The larvae were surfacesterilized with $75 \%$ ethanol for $60 \mathrm{~s}$ and rinsed three times with sterilized deionized water. The dissection was performed on ice under sterile conditions. Twenty larval guts were pooled as one sample and three samples were established for each population. Isolated gut contents were immediately placed in precooled Eppendorf tubes and homogenized with $1 \mathrm{~mL}$ sterile $1 \times$ phosphate buffered saline (PBS, pH 7.2). All the samples were divided into two parts, one for culture-dependent method and the other for amplicon sequencing. Samples used for amplicon sequencing were flash-frozen with liquid nitrogen and homogenized with a pestle to extract DNA.

\subsection{Culture-Dependent Microbial Communities}

For cultivation experiment, the gut content suspension was diluted into $10^{-4}, 10^{-5}$, and $10^{-6}$ dilution series with sterile $1 \times$ PBS, and an aliquot of $100 \mu \mathrm{L}$ of the suspension was spread on plates of LB (Lucia-Bertani), TSB (Trypticase Soy Broth), GSA (Gause's synthetic agar; HKM, Guangzhou, China), HIA (Heart infusion agar; BD, Baltimore, Maryland, USA), and PPDA $[18,43]$ to isolate the bacteria and fungi. Twelve plates were set up for each treatment. All the plates were sealed with parafilm (BEMIS, Ninah, Wisconsin, USA) and then cultured in the dark condition at $13{ }^{\circ} \mathrm{C}$ and $25^{\circ} \mathrm{C}$, respectively.

The growing colonies of bacteria and fungi on the plates were screened on the basis of colony appearance and further purified by quadrant streaking on LB or PPDA plates, respectively. To identify the microbe isolates, the prokaryotic V4 region of $16 \mathrm{~S}$ rRNA was amplified with primers 27F (5'-AGAGTTTGATCCTGGCTCAG-3')/1492R (5'-TACGGYTA CCTTGTTACGACTT-3') for bacteria and the ITS2 region of ITS gene was amplified with ITS5 (5'-GGAAGTAAAAGTCGTAACAAGG-3')/ITS4 (5'-TCCTCCGCTTATTGATATGC-3') for fungi, respectively. The PCR products were separated by electrophoresis and the bands were cut from the gels for purification, and then sequenced in Sangon Biotech (Shanghai) Co., Ltd. (Guangzhou, China). Sequence analysis was performed using nucleotide blast http:/ / www.ncbi.nlm.nih.gov/ (accessed on 1 November 2019). The representative sequences of each bacterial and fungal species were submitted to GenBank https: / www.ncbi.nlm.nih. gov/genbank/ (accessed on 2 February 2020) under accession numbers MW555179-MW555204 and MW555208-MW555221, respectively. 


\subsection{Culture-Independent Microbial Communities}

Total DNA was extracted from each pool using the QIAamp DNA Stool Mini Kit (Qiagen, Hilden, Germany) according to the manufacturer's instructions. 16S rRNA and ITS gene amplicons of the gut samples were produced and sequenced using an Illumina Nova 6000 platform (Guangdong Magigene Biotechnology Co., Ltd. Guangzhou, China). Briefly, the V5-V6 region of the 16S rRNA gene was amplified using primers 799F (5'AACMGGATTAGATACCCKG-3')/1193R (5'-ACGTCATCCCCACCTTCC-3') with $12 \mathrm{bp}$ barcode. The ITS1 region of the fungal ITS gene was amplified using the primers BD-ITS1F (5'-GGAAGTAAAAGTCGTAACAAGG-3')/ITS2-2043R (5'-GCTGCGTTCTTCATCGATGC$\left.3^{\prime}\right)$ with 12 bp barcode.

The sequencing data were qualified using Fastp (version 0.14.1). To obtain the pairedend clean reads, the primers were removed by using cutadapt software (https:/ / github. $\mathrm{com} / \mathrm{marcelm} /$ cutadapt/ (accessed on 2 March 2020). Paired-end clean reads were merged using usearch -fastq_mergepairs (V10http://www.drive5.com/usearch/ (accessed on 2 March 2020). Raw tags were merged when at least 16 bp overlap the read generated from the opposite end of the same DNA fragment, with the maximum mismatch allowed in overlap region being $5 \mathrm{bp}$. Clean Tags were generated after removal of barcodes and primers. The resulting high-quality reads were clustered into operational taxonomic units (OTU) by usearch-sintax (set the confidence threshold to default to $\geq 0.8$ ) using SILVA (V119, http:/ / www.arb-silva.de (accessed on 2 March 2020) for bacteria and UNITE 252 V7.0, http:/ / unite.ut.ee/index.php (accessed on 12 March 2020) for fungi. After removal of the OTU and its Tags, which were annotated as chloroplasts or mitochondria (16S amplicons) and not annotated to the kingdom level, the final OTU taxonomy synthesis information table was obtained.

For each sample, alpha diversity was applied to estimate complexity of species for a sample by calculating bacterial and fungal alpha diversity indices, including richness, chao 1, and Simpon's index. All these indices were calculated with usearch-alpha_div (V10, http:/ / www.drive5.com/usearch/ accessed on 2 March 2020) and calculated rarefaction curve and rank abundance separately. The differences between groups were analyzed by alpha diversity indices using Student's t-test for two groups and Kruskal Wallis for more than two groups $(p=0.05)$ with $\mathrm{R}$ software. Heat maps at the phylum, family, and genus levels among the six groups were generated with $\mathrm{R}$ using ampvis2 package [44].

Beta diversity was measured by Principal Coordinate Analysis (PCoA) based on BrayCurtis distance matrixes and was displayed by vegan package in $\mathrm{R}$ Software. In addition, unweighted pair-group method with arithmetic means (UPGMA) clustering analysis was also performed to interpret the distance matrix using average linkage. To analyze the difference of community structure between groups, permutational multivariate analysis of variance (Permanova) statistical analyses were conducted based on Bray-Curtis distance matrixes with 999 permutations using two non-parametric analyses including analysis of similarity (Anosim) and non-parametric multivariate analysis of variance (Adonis) of vegan and pegas package in $R$ software.

To identify microbes accounting for the effects of geographic locations and altered habitat conditions, the linear discriminatory analysis (LDA) effect size (LEfSe) algorithm was used to compare the differential abundances of bacteria and fungi among groups at family and genus levels using LEfSe software [45]. Non-parametric factorial Kruskal Wallis sum rank test was used to identify taxa with significant difference in abundance among groups $(p<0.05)$. Then, Wilcoxon rank sum test was used to investigate biological consistency among subgroups. Finally, LDA was used to estimate the impact of each selected taxon. Only those taxa with more than four orders of magnitude (LDA score $>4$ ) were considered in this study.

All obtained 16S and ITS amplicon data have been deposited in the Sequence Read Archive (SRA) under accession number PRJNA698401. 


\section{Results}

\subsection{Molecular Identification of the Ghost Moth Populations}

The information on six groups (W.SD, W.GG, W.XJ, A.SD, A.GG, and A.XJ) including sampling altitude (masl, meters above sea level), environmental temperature, major diet, and location were presented in Table 1. Larvae derived from Xiaojin County (W.XJ and A.XJ) were identified as Thitarodes xiaojinensis by the mitochondrial cytochrome $b$ sequence. The phylogenetic tree of the ghost moths from the above three locations constructed by the mitochondrial cytochrome $b$ gene showed that population W.SD was closely related to population W.GG (Figure S2).

Table 1. Group information of the 16S rRNA and ITS gene analyses.

\begin{tabular}{|c|c|c|c|c|}
\hline Group Name ${ }^{1}$ & Altitude (Masl) & Environmental Temperature ${ }^{2}$ & Major Diet ${ }^{3}$ & Sampling Location \\
\hline W.SD (W) & $\sim 4128$ & Wild, $-20 \sim 10{ }^{\circ} \mathrm{C}$ & $\begin{array}{l}\text { plant roots of alpine } \\
\text { meadow }\end{array}$ & $\begin{array}{c}\text { Shade Town, Kangding City, Ganzi } \\
\text { Tibetan Autonomous Prefecture, } \\
\text { Sichuan Province, China }\end{array}$ \\
\hline W.GG (W) & $\sim 3958$ & Wild, $-20 \sim 10{ }^{\circ} \mathrm{C}$ & $\begin{array}{l}\text { plant roots of alpine } \\
\text { meadow }\end{array}$ & $\begin{array}{c}\text { Hailuogou, Gongga mountain, Moxi } \\
\text { Town, Luding County, Ganzi Tibetan } \\
\text { Autonomous Prefecture, Sichuan } \\
\text { Province, China }\end{array}$ \\
\hline W.XJ (W) & $\sim 3823$ & Wild, $-20 \sim 10{ }^{\circ} \mathrm{C}$ & $\begin{array}{l}\text { plant roots of alpine } \\
\text { meadow }\end{array}$ & $\begin{array}{c}\text { Xiaojin County, Aba Tibetan and Qiang } \\
\text { Autonomous Prefecture, Sichuan } \\
\text { Province, China }\end{array}$ \\
\hline A.SD (A) & $\sim 43$ & Artificial rearing at $8^{\circ} \mathrm{C}$ & roots of $P$. anserina & $\begin{array}{l}\text { Haizhu District, Guangzhou City, } \\
\text { Guangdong Province, China }\end{array}$ \\
\hline A.GG (A) & $\sim 43$ & Artificial rearing at $8{ }^{\circ} \mathrm{C}$ & roots of $P$. anserina & $\begin{array}{l}\text { Haizhu District, Guangzhou City, } \\
\text { Guangdong Province, China }\end{array}$ \\
\hline A.XJ (A) & $\sim 43$ & Artificial rearing at $8{ }^{\circ} \mathrm{C}$ & roots of $P$. anserina & $\begin{array}{l}\text { Haizhu District, Guangzhou City, } \\
\text { Guangdong Province, China }\end{array}$ \\
\hline
\end{tabular}

${ }^{1}$ A.SD, A.GG and A.XJ larvae were artificially reared in the laboratory from the pupae of W.SD, W.GG, and W.XJ, respectively; ${ }^{2}$ The environment temperature is cited from the reference [46]; ${ }^{3}$ More than 100 species in 19 families of host plants for the wild ghost moth larvae [47].

\subsection{Culture-Dependent Communities}

Overall, 26 bacterial species belonging to 21 genera and 14 fungal species belonging to 12 genera were identified from the gut of the ghost moth larvae from the six populations. At the phylum level, 11, 5, 9, and 1 species of 26 bacterial species were assigned to Proteobacteria, Firmicutes, Actinobacteria, and Bacteroidetes, respectively. For fungi, 9, 3, and 2 species were assigned to Ascomycota, Basidiomycota, and Mucoromycota, respectively. Four culturable bacterial species including Carnobacterium maltaromaticum, Rahnella aquatilis, Pseudomonas sp., and Streptomyces sp. were considered as shared species, among which C. maltaromaticum was the most abundant bacterial species cultivated from both the wild and laboratory-reared samples (Table 2).

Compared with the laboratory-reared larvae, more colonies of $C$. maltaromaticum were detected from the wild ghost moth larvae. Raoultella terrigena was shared in the wild samples collected from the three different locations but not detected in the laboratoryreared populations. Agromyces sp. and Pseudoclavibacter sp. were only detected in the wild larvae from SD population, while Oerskovia sp. and Pantoea sp. were only detected from GG population and Buttiauxella sp. were only detected from XJ population. Similarly, more fungal species appeared from the wild larvae and no fungal species were shared in all six populations (Table 2).

The percentages of the microbe species obtained from the above five different media at two different temperatures are shown in Table S1. Generally, more bacterial and fungal species were isolated from the plates containing TSB and PPDA media at $23^{\circ} \mathrm{C}$. 
Table 2. Bacterial and fungal species in the gut of the wild and laboratory-reared Thitarodes populations by culture-dependent method.

\begin{tabular}{|c|c|c|c|c|c|c|c|c|}
\hline \multirow{2}{*}{ Category } & \multirow{2}{*}{ Microbe Species } & \multirow{2}{*}{ Phylum } & \multicolumn{6}{|c|}{ Population Sampled } \\
\hline & & & W.SD & W.GG & W.XJ & A.SD & A.GG & A.XJ \\
\hline \multirow[t]{26}{*}{ Bacteria } & Acinetobacter lwoffii & Proteobacteria & + & & & & & + \\
\hline & Aeromonas sp. & Proteobacteria & & + & ++ & & & \\
\hline & Agromyces sp. & Actinobacteria & + & & & & & \\
\hline & Arthrobacter sp. & Actinobacteria & + & & & + & + & + \\
\hline & Bacillus mycoides & Firmicutes & & & + & & & \\
\hline & Bacillus sp. & Firmicutes & & + & & + & & + \\
\hline & Buttiauxella sp. & Proteobacteria & & & + & & & \\
\hline & $\begin{array}{l}\text { Carnobacterium } \\
\text { maltaromaticum }\end{array}$ & Firmicutes & ++++ & ++++ & ++++ & +++ & +++ & +++ \\
\hline & Chryseobacterium sp. & Bacteroidetes & + & & + & + & + & + \\
\hline & Enterococcus sp. & Firmicutes & & ++ & & & & + \\
\hline & Glutamicibacter sp. & Actinobacteria & + & & & + & & \\
\hline & $\begin{array}{l}\text { Microbacterium } \\
\text { foliorum }\end{array}$ & Actinobacteria & & + & & & & \\
\hline & Microbacterium sp. & Actinobacteria & + & & + & + & + & ++ \\
\hline & Oerskovia sp. & Actinobacteria & & + & & & & \\
\hline & Pantoea sp. & Proteobacteria & & + & & & & \\
\hline & Pseudomonas sp. & Proteobacteria & + & + & + & ++ & + & + \\
\hline & Pseudomonas fragi & Proteobacteria & + & & + & + & & + \\
\hline & Pseudoclavibacter sp. & Actinobacteria & + & & & & & \\
\hline & Rahnella aquatilis & Proteobacteria & + & + & + & + & + & + \\
\hline & Raoultella terrigena & Proteobacteria & + & + & + & & & \\
\hline & Rhodococcus sp. & Actinobacteria & + & & + & & & + \\
\hline & Serratia fonticola & Proteobacteria & & + & + & & & ++ \\
\hline & Serratia plymuthica & Proteobacteria & + & + & & + & + & \\
\hline & $\begin{array}{c}\text { Serratia } \\
\text { proteamaculans }\end{array}$ & Proteobacteria & & + & & & + & \\
\hline & Staphylococcus sp. & Firmicutes & & & + & + & + & + \\
\hline & Streptomyces sp. & Actinobacteria & + & + & + & + & + & + \\
\hline \multirow[t]{16}{*}{ Fungi } & Apiotrichum sp. & Basidiomycota & & + & & & & \\
\hline & Aspergillus sp. & Ascomycota & + & & + & & & + \\
\hline & Candida sp. & Ascomycota & ++ & ++ & & + & + & \\
\hline & Chaetomium sp. & Ascomycota & & + & + & & & \\
\hline & Cladosporium sp. & Ascomycota & + & + & + & + & & \\
\hline & Mucor hiemalis & Mucoromycota & & + & & & & \\
\hline & Mucor racemosus & Mucoromycota & + & & & & + & \\
\hline & Nectriaceae & Ascomycota & & + & + & & & + \\
\hline & Penicillium polonicum & Ascomycota & & & + & & & \\
\hline & Penicillium sp. & Ascomycota & + & & + & & & + \\
\hline & Rhodotorula sp. & Basidiomycota & & + & & & & \\
\hline & Trichoderma sp. & Ascomycota & & + & & & & \\
\hline & Sporocadaceae & Ascomycota & & & + & & & \\
\hline & $\begin{array}{l}\text { Sterigmatomyces } \\
\text { halophilus }\end{array}$ & Basidiomycota & & + & & + & & \\
\hline & Bacterial species & & 15 & 14 & 14 & 12 & 10 & 14 \\
\hline & Fungal species & & 5 & 9 & 7 & 3 & 2 & 3 \\
\hline
\end{tabular}

W.SD, W.GG, and W.XJ larval populations were collected from the high-altitude alpine meadow in Sichuan, China. A.SD, A.GG, and A.XJ larvae were artificially reared in the laboratory from the pupae of W.SD, W.GG, and W.XJ populations, respectively.,$+ \leq 100$ colonies detected in the plates;,$++>100 \leq 1000$ colonies detected in the plates;,$+++>1000 \leq 2500$ colonies detected in the plates;,$++++>2500$ colonies detected in the plates.

\subsection{Culture-Independent Communities}

A total of 1,571,246 high-quality clean reads of bacteria (16S rRNA), and of 1,578,989 high-quality clean reads of fungi (ITS) were produced from the six populations with 3 replicates for each population (Table S2), indicating high quality sequencing. Each sample contained at least 60,000 effective sequences for $16 \mathrm{~S}$ and ITS amplicons (Figure S3). The rarefaction curves for all samples showed that the sequence depths were reliable for both bacterial and fungal identification in each sample (Figure S4). 


\subsubsection{General Pattern of the Gut Microbiota of the Wild Ghost Moth Populations}

In the total dataset, most of the bacteria were identified as Firmicutes $(98.79,15.14$ and $35.75 \%$ in the gut of the wild SD, GG and XJ, respectively), followed by Proteobacteria $(0.78,84.68$ and $63.63 \%$ in the wild SD, GG and XJ) and to a lesser extent, Bacteroidetes and Actinobacteria (Figure 1A). At the family level, the most abundant bacterial taxa in the wild SD were Carnobacteriaceae. However, the wild GG and XJ were dominated by Enterobacteriaceae, followed by Carnobacteriaceae (Figure 1B). At the genus level, the wild SD bacterial community was dominated by Carnobacterium, while the wild GG was dominated by Serratia, f-Enterobacteriaceae_OTU_4, Carnobacterium, Enterococcus, and $f$-Enterobacteriaceae_OTU_55. The most frequently occurring genera from the wild XJ were Serratia, Carnobacterium, f-Enterobacteriaceae_OTU_4,f-Enterobacteriaceae_OTU_7, and Aeromonas (Figure 1C).
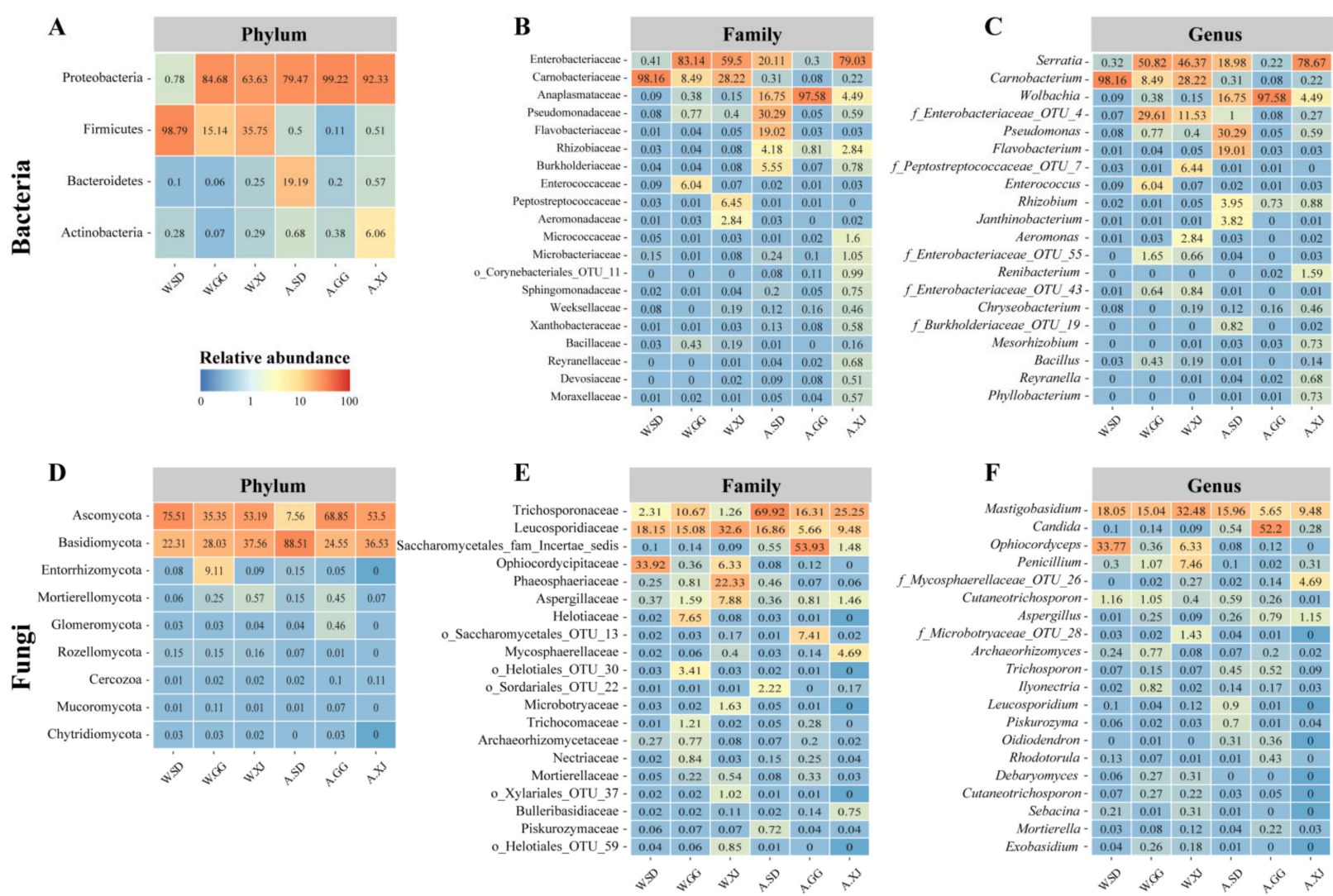

Figure 1. The bacterial and fungal communities of the wild and laboratory-reared ghost moth larvae. Heatmaps of the bacterial (A) and fungal (B) top phyla, bacterial (C) and fungal (D) top 20 family, and bacterial (E) and fungal (F) top 20 genera based on the relative abundance of OTUs in all samples. Relative abundance is the mean of replicate samples.

For fungi, the wild SD and XJ populations were dominated by Ascomycota (Comprising 75.51 and $53.19 \%$, respectively), followed by Basidiomycota (Comprising 22.31 and $37.56 \%$, respectively), and the wild GG population was dominated by Ascomycota (35.35\%), Basidiomycota (28.03\%), and Entorrhizomycota (9.11\%) (Figure 1D). At the family level, the most abundant fungal taxa in the three wild populations were different, Ophiocordycipitaceae, Leucosporidiaceae, and Trichosporonaceae in the wild SD; Leucosporidiaceae, Trichosporonaceae, Helotiaceae, and Aspergillaceae in the wild GG; and Leucosporidiaceae, Phaeosphaeriaceae, Aspergillaceae, Ophiocordycipitaceae, Microbotryaceae, and Trichosporonaceae in the Wild XJ (Figure 1E). The most frequently occurring genera of the wild SD population were Ophiocordyceps, Mastigobasidium, and Cutaneotrichosporon. The most frequently occurring genera of the wild GG population were Mastigobasidium, Penicillium, Cutaneotrichosporon, Ilyonectria, and Archaeorhizomyces. The most frequently occurring 
genera of the wild XJ population were Mastigobasidium, Penicillium, and Ophiocordyceps (Figure 1F).

The Richness and Chao1 indices of the gut bacteria in the wild XJ were significantly higher than those in the wild SD and GG. The Simpson indices of the gut bacteria and fungi in the wild SD were significantly higher than those in the wild GG and the wild XJ, which indicated that the bacterial and fungal diversities of the wild SD were significantly lower than those of the wild GG and the wild XJ (Figure 2 and Table S3). However, the ANOSIM and Adonis analyses revealed no significant microbial community difference in the wild ghost moth collected from different geographic locations (Figure 3 and Table S4).
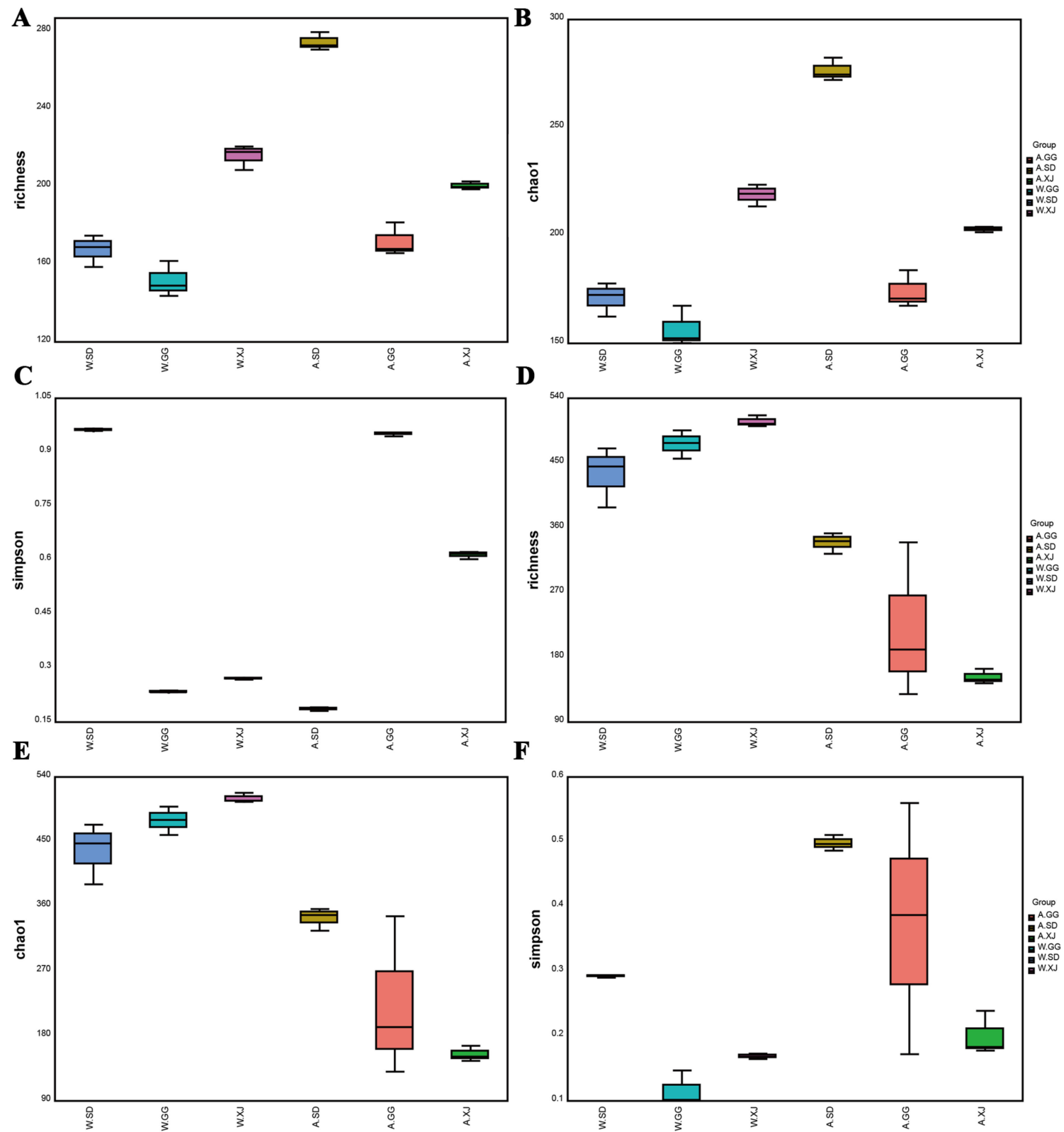

Figure 2. The alpha diversity of the gut microbial composition. Richness, Chao1, and Simpson indices of bacteria (A-C) and fungi (D-F). Richness means the number of OTUs determined with 16S rRNA gene (Bacteria) or ITS gene (Fungi) based on DNA extracted from populations; Chao 1 means the total number of OTUs estimated by infinite sampling, and a higher number indicates a higher richness [48]; Simpson's index measures community evenness, and index increases as diversity decreases [49]. 
A

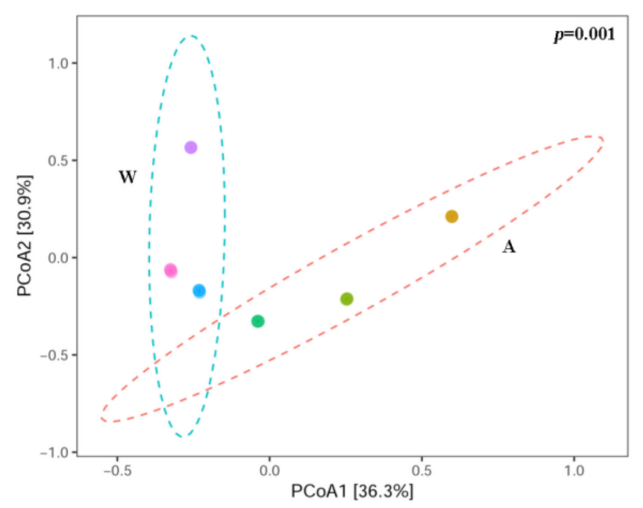

B

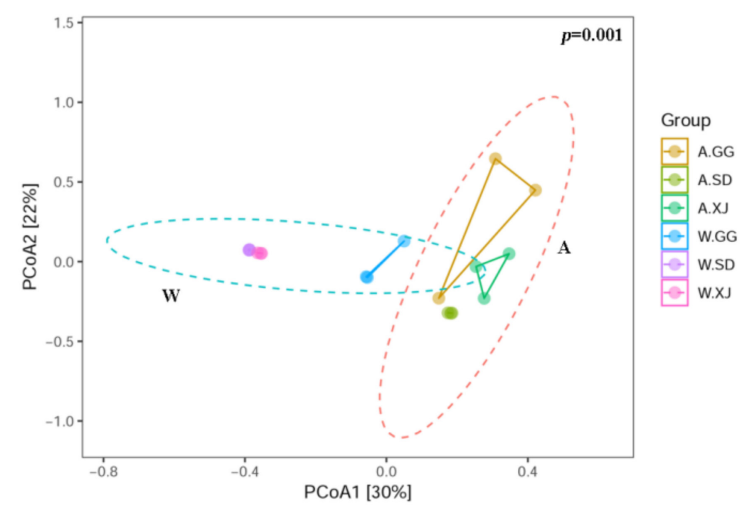

Figure 3. PCoA visualization using the Bray-Curtis dissimilarity measurement separating samples. PCoA analysis based on Bray-Curtis distance matrix for bacterial (A) and fungal (B) communities of all samples.

3.3.2. Comparison of the Gut Microbial Community Diversity between Insect Populations

A comparison of alpha diversity indices between ghost moth populations was presented in Figure 2 and the significance was detected by turkey method. For bacteria, there were significant differences among the laboratory-reared ghost moths from SD, GG, and XJ populations (A.SD versus A.GG, A.SD versus A.XJ, and A.GG versus A.XJ). The bacterial richness and diversity of the laboratory-reared ghost moth from SD population were significantly higher than those from GG and XJ populations (Figure 2A-C and Table S3). In addition, the laboratory-reared ghost moth was notably dissimilar with the wild ghost moth (A.SD versus W.SD, A.GG versus W.GG, and A.XJ versus W.XJ). Artificial rearing of the larvae in the laboratory significantly increased the bacterial richness and diversity in SD population, but significantly decreased the bacterial diversity in GG and XJ population. For fungi, there was a significant difference between the laboratory-reared ghost moths from SD and XJ populations (A.SD versus A.XJ), but no detectable significant differences were found between A.SD and A.GG or A.GG and A.XJ. Compared with the wild ghost moth, artificial rearing significantly decreased the fungal richness and diversity in SD and XJ populations (A.SD versus W.SD and A.XJ versus W.XJ). However, no detectable significant difference was found between the laboratory-reared and wild ghost moth from GG population (A.GG versus W.GG) (Figure 2D-F and Table S3).

Similarities in the bacterial and fungal community compositions between populations were compared by PCoA based on the Bray-Curtcis index. The ANOSIM and Adonis analyses revealed significant microbial community difference between the wild and laboratory-reared samples (Figure 3 and Table S4). However, the wild and laboratoryreared populations from the same location tended to separate from each other for bacterial community diversity but the difference was not significant (Figure 3A and Table S4). For fungal community diversity, the wild SD and XJ populations were slightly separated from the laboratory-reared SD and XJ populations, respectively (Figure 3B and Table S4). Nevertheless, one wild GG sample (W.GG3) was separated from the other wild GG samples, and one laboratory-reared GG sample (A.GG3) was also separated from the other laboratoryreared GG samples. These differences were also revealed by UPMA clustering (Figure 4). The separation of sample W.GG3 from samples W.GG1 and W.GG2 was due to increased abundance of Helotiaceae and Saccharomycetales, while the separation of sample A.GG3 from samples A.GG1 and A.GG2 was due to decreased abundance of Candia and increased abundance of OTUs belonging to Mastigobasidium and Trichosporonaceae. 
$\mathbf{A}$
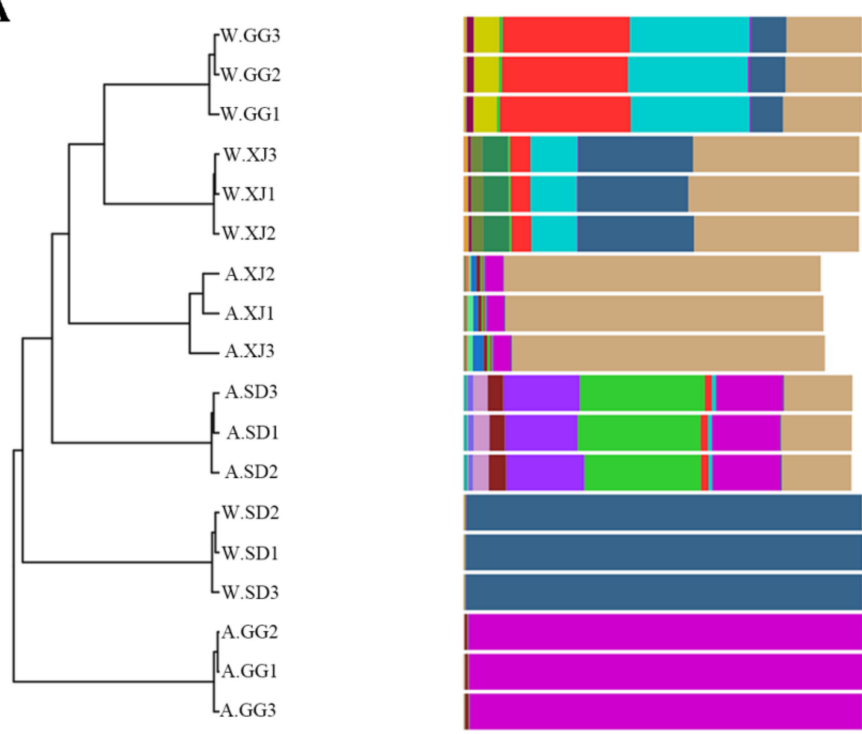

OTU_18:g:Mesorhizobium

OTU_19:f:Burkholderiaceae

OTU_17:g:Chryseobacterium

OTU_11:0:Corynebacteriales

OTU_43:f:Enterobacteriaceae

OTU_27:c:Gammaproteobacteria

OTU_12:g:Arthrobacter

OTU_55:f:Enterobacteriaceae

OTU_13:g:Aeromonas

OTU_10:g:Janthinobacterium

OTU_9:g:Rhizobium

OTU_7:f:Peptostreptococcaceae

OTU_122:g:Enterococcus

OTU_8: :Flavobacterium

OTU_5:g:Pseudomonas

OTU_6:g:Serratia

OTU_4:f:Enterobacteriaceae

OTU_3:g:Wolbachia

OTU_1:s:Carnobacterium maltaromatium

OTU_2:g:Serratia

\section{B}
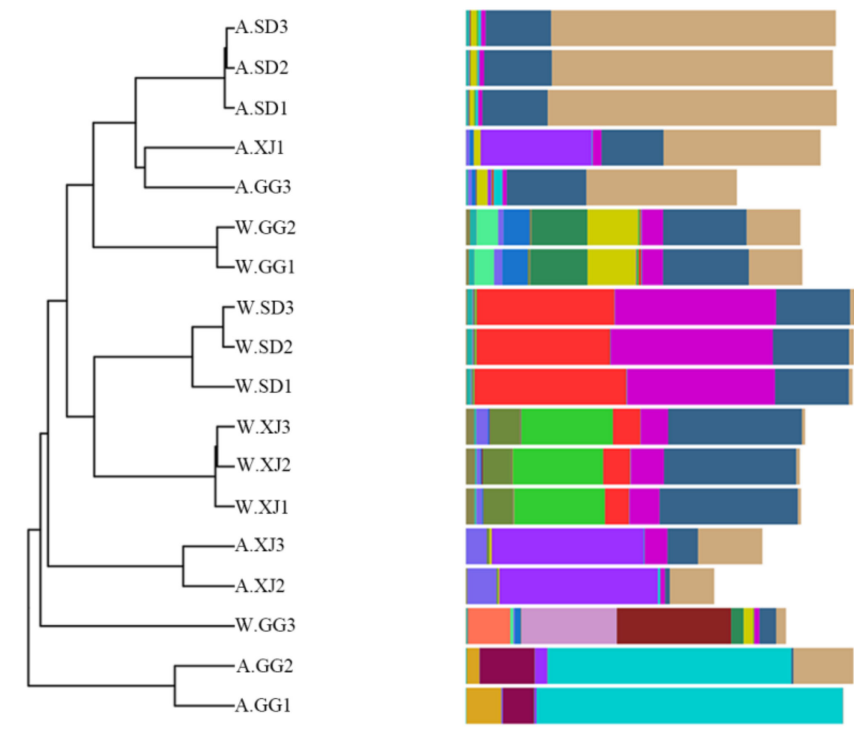

OTU_23:k:Fungi

OTU_20:g:Cutaneotrichosporon

OTU_30:0:Helotiales

OTU 21:c:Pezizomycotina_cls_Incertae_sedis

OTU_16:s:Candida railenensis

OTU_17:k:Fungi

OTU_15:p:Ascomycota

OTU 13:0:Saccharomycetales

OTU_11:g:Penicillium

OTU_12:f:Helotiaceae

OTU 10:c:Entorrhizomycetes

OTU_14:k:Fungi

OTU 9:k:Fungi

OTU_8:p:Ascomycota

OTU_7:f:Phaeosphaeriaceae

OTU_5:g:Ophiocordyceps

OTU_6:g:Candida

OTU_3: p:Ascomycota

OTU_2:g:Mastigobasidium

OTU_1: f:Trichosporonaceae

Figure 4. Clustering analysis of beta-diversity based on the Bray-Curtis distance matrix for bacterial (A) and fungal (B) communities of all samples by UPGMA method.

\subsubsection{Differential Microbes among Insect Populations}

Based on the significant differences among the samples by using linear discriminant analysis (LDA), 9 genera and 10 families of bacteria and 4 genera and 8 families of fungi were significantly enriched in different populations (Figure 5). The relative abundance of each selected genus was presented in Figure 6. 


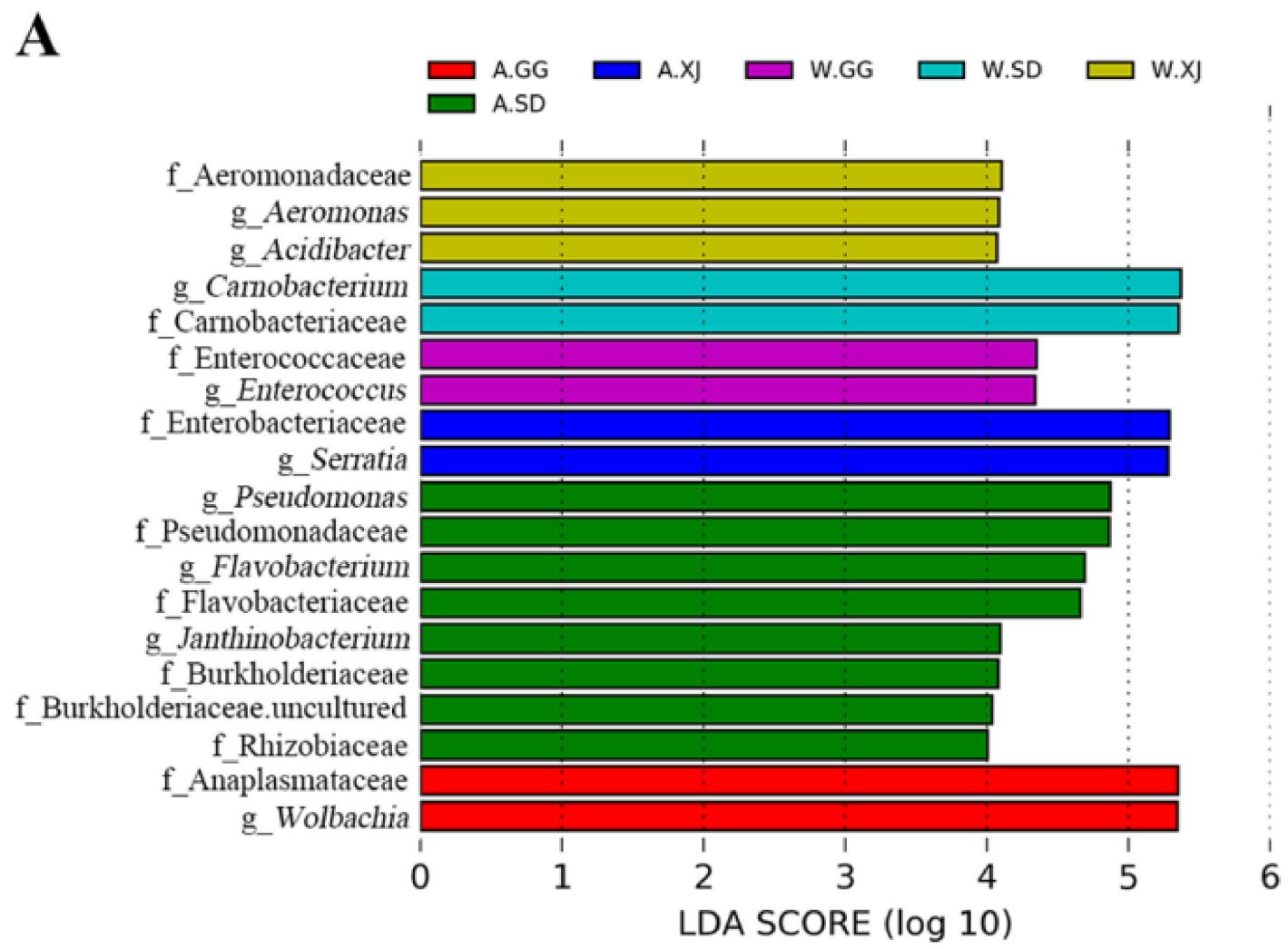

\section{B}

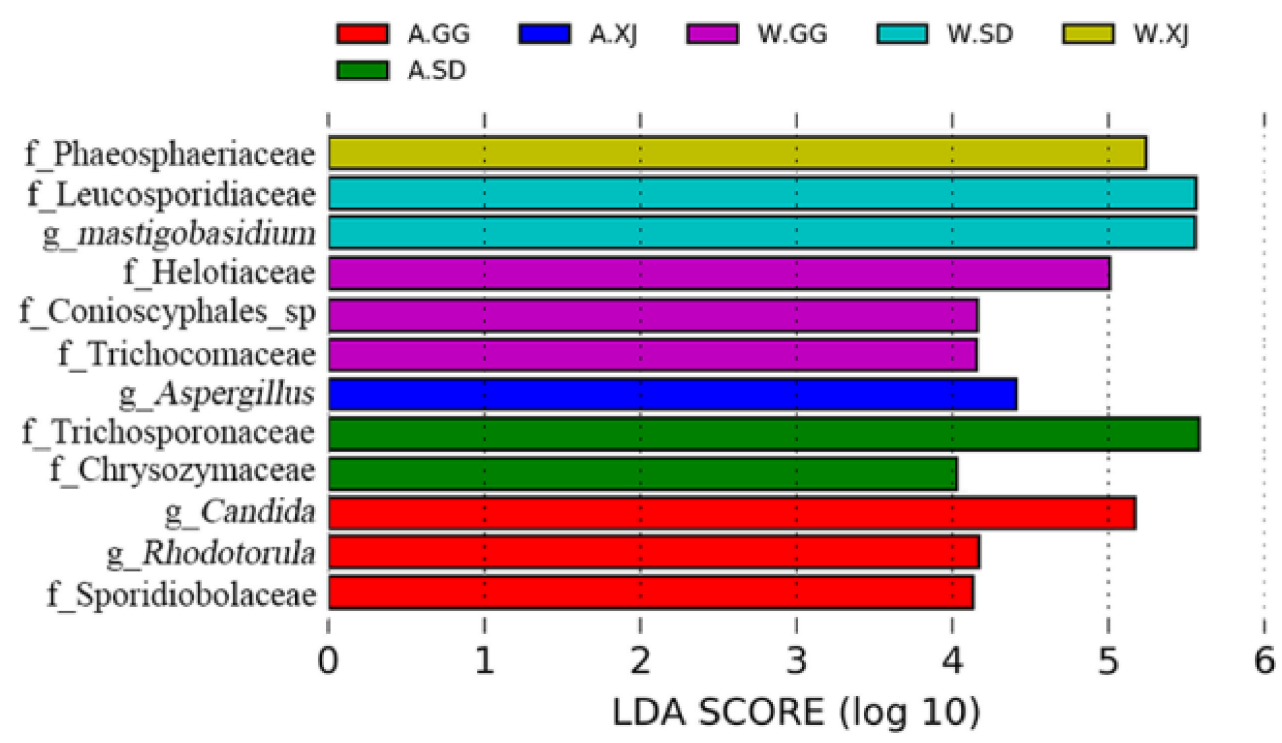

Figure 5. Differences in bacterial (A) and fungal (B) taxa among groups determined by linear discriminative analysis effect size (LEfSe). LDA scores could be interpreted as the degree of difference in relative abundance. Abbreviation: $g_{-}$, genus and f_, family. 
A

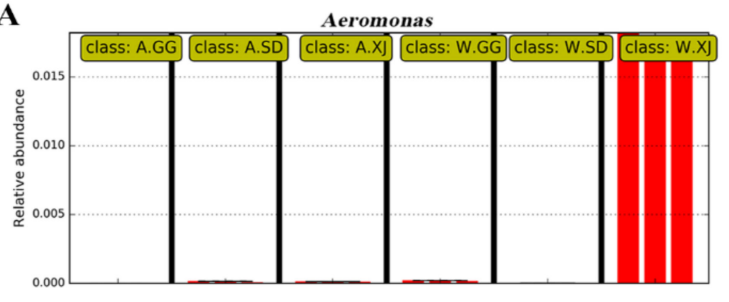

Carnobacterium

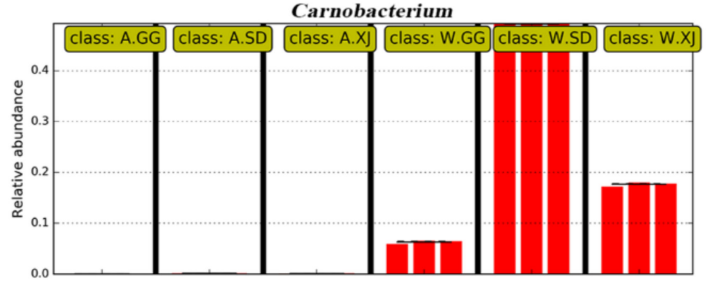

$\mathbf{B}$
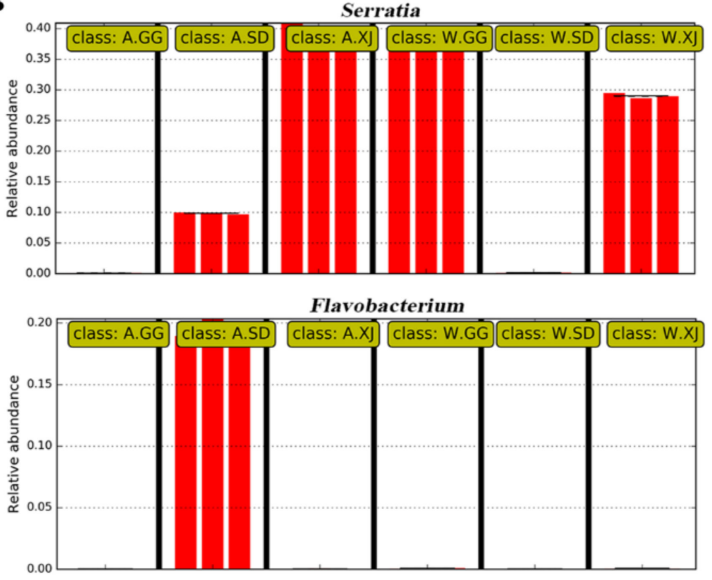

Wolbachia

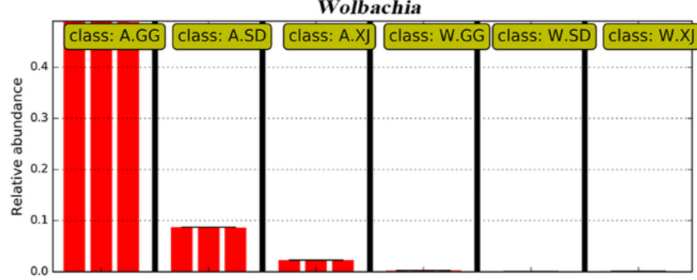

C
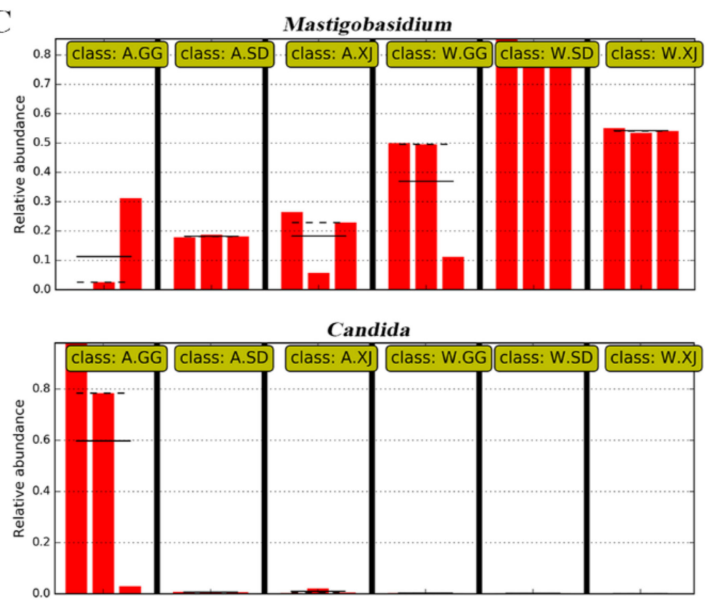
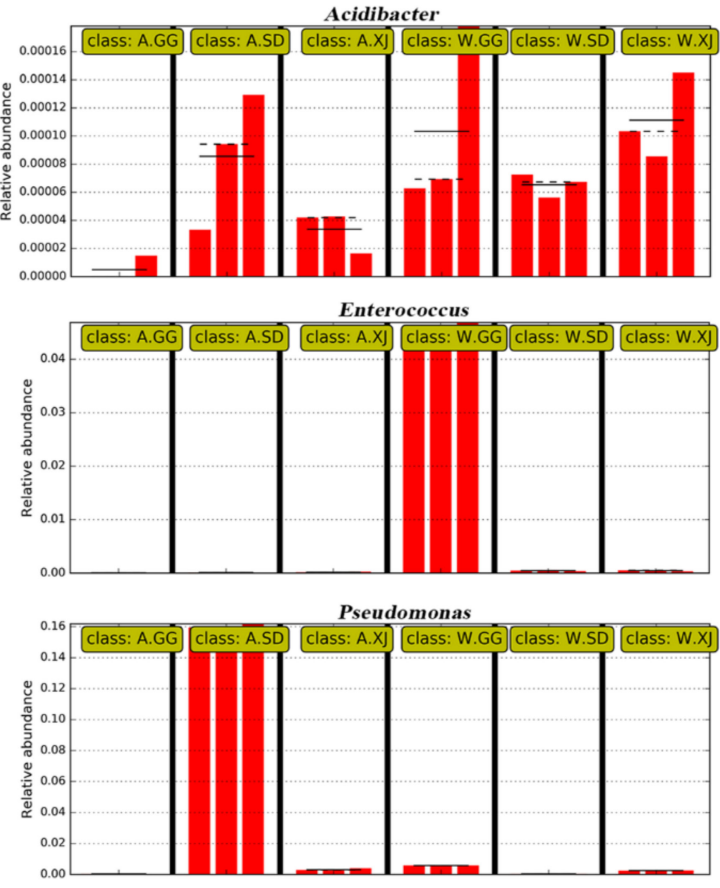

Janthinobancterium

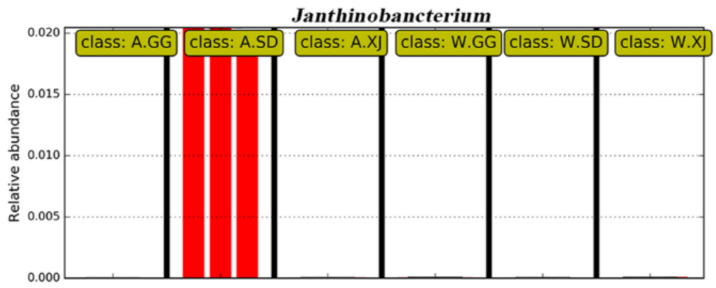

Aspergillus

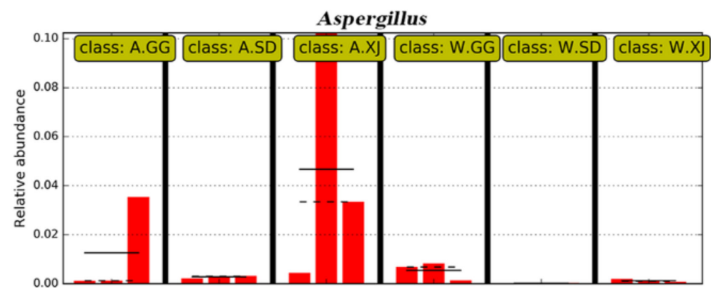

Rhodotorula

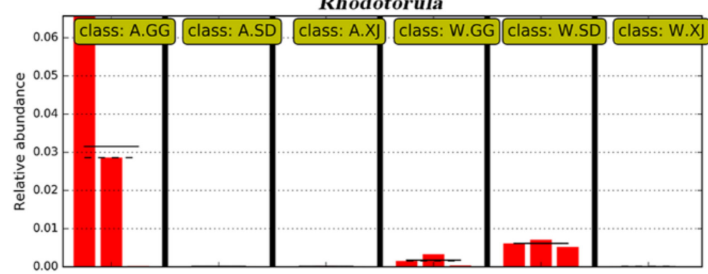

Figure 6. Relative abundances of differential microbes at the genus level selected by LEfSe analysis among populations. The straight lines indicate the mean. The dotted lines indicate the median. (A) Dominant bacteria in the gut of the wild ghost moth; (B) Dominant bacteria in the gut of the laboratory-reared ghost moth larvae; (C) Dominant fungal in the gut of the wild and laboratory-reared ghost moth larvae. 
When compared with the wild ghost moth, the gut bacteria of the laboratory-reared SD population showed increased abundance of genera Pseudomonas, Flavobacterium, and Janthinobacterium and families Pseudomonadaceae, Flavobacteriaceae, Burkholderiaceae, and Rhizobiaceae. The laboratory-reared GG population had a significant higher abundance of genus Wolbachia and family Anaplasmataceae, while the laboratory-reared XJ population showed increased abundance of genus Serratia and family Enterobacteriaceae (Figure 5A). For fungi, the laboratory-reared SD population showed increased abundance of families Trichosporonaceae and Chrysozymaceae, while the laboratory-reared GG population showed increased abundance of genera Candida and Rhodotorula and family Sporidiobolaceae. The laboratory-reared XJ population had a higher abundance of genus Aspergillus (Figure 5B).

A lot of fungal pathogens could infect and kill the larvae, and the most fatal pathogen is Isaria farinosa (formerly Paecilomyces farinosus) based on the identification of isolates from the ghost moth cadaver killed by microbes during the artificial rearing of the ghost moth (Table S5). When checking the relative abundance of these fungal pathogens, Ophiocordyceps, which infected the ghost moth larvae to form the Chinese cordyceps, showed a higher relative abundance in the wild larvae, especially in the wild SD population than that in the laboratory-reared larvae (Figure 7A). Another entomopathogenic fungus Beauveria bassiana was present in the gut of both the wild and laboratory-reared larvae from SD population, but its abundance was very low. The entomopathogenic fungus Metarhizium showed increased abundance in the wild larvae from XJ population (Figure 7B). The wound pathogenic fungus Mucor and the saprophytic fungus Penicillium were also present in the gut of the wild and laboratory-reared ghost moths (Figure 7C,D). However, the most fatal entomopathogenic fungus $I$. farinosa was not detected in the gut of both the wild and laboratory-reared larvae, which was further demonstrated by PCR amplification using the specific primers [50] of I. farinosa (Figure S5).

A

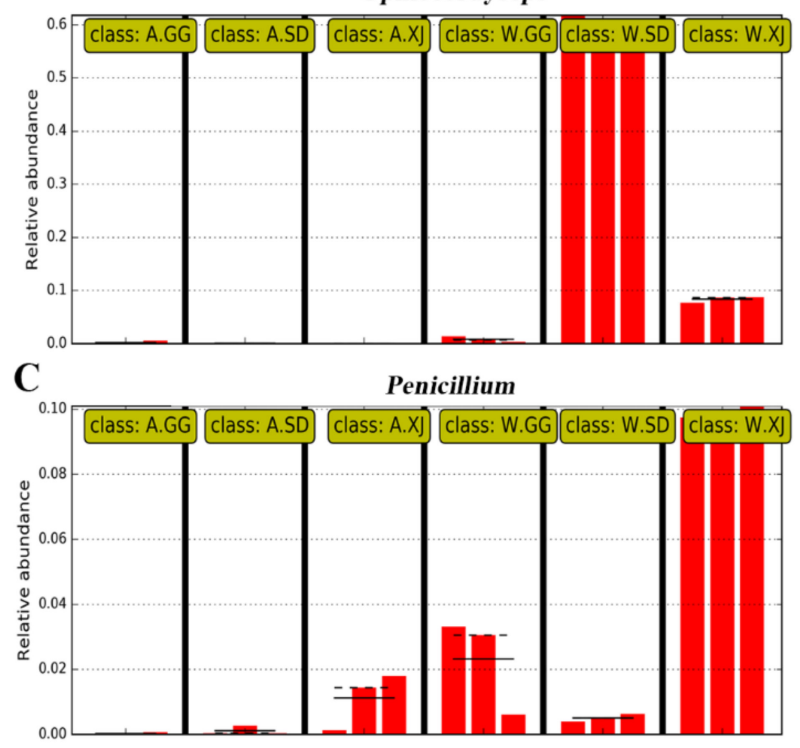

B

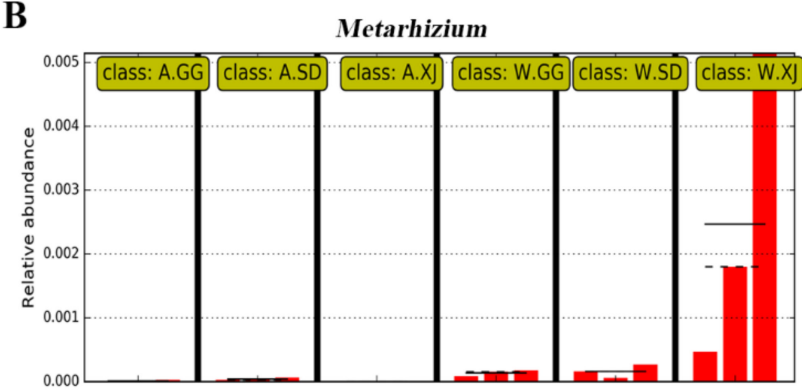

D

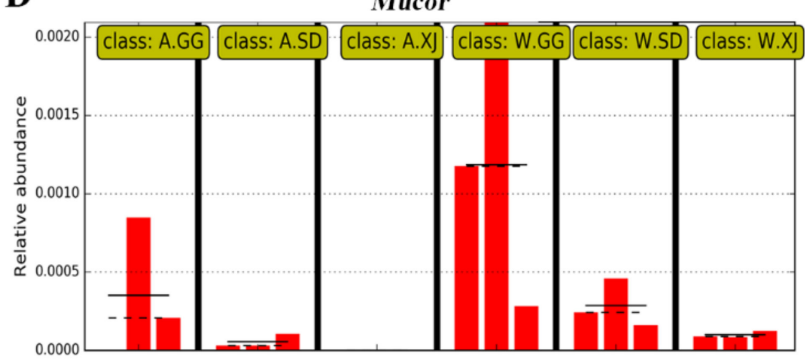

Figure 7. Relative abundance of the fungus isolated from the mummified laboratory-reared ghost moth larvae. (A) Relative abundance of Ophiocordyceps; (B) Relative abundance of Metarhizium; (C) Relative abundance of Penicillium; (D) Relative abundance of Mucor.

\section{Discussion}

By employing a culture-dependent and -independent 16S rRNA and ITS gene highthroughput sequencing analyses, comprehensive information was obtained on the gut bacterial and fungal community in the ghost moth larvae of three different geographic locations from high-altitude on Tibet plateau and from low-altitude laboratory. The discov- 
ery of apparent microbial community shifts between the wild and laboratory-reared ghost moth larvae, many opportunistic pathogenic bacteria and fungi in the gut of the laboratoryreared larvae, and the enriched dominant bacteria in the wild ghost moth provide new insights to improve the effectiveness of the laboratory-reared Thitarodes hosts of O. sinensis for the cultivation of the Chinese cordyceps.

The culture-dependent method resulted in the isolation of 21 bacterial genera and 12 fungal genera from six ghost moth populations (Table 2). Compared with at most eight bacterial genera and three fungal genera isolated from the gut of the wild T. gonggaensis larvae $[39,40,51]$, more bacteria and fungi were isolated in the present study, and seven bacterial genera Acinetobacter, Aeromomonas, Bacillus, Carnobacterium, Pantoea, Pseudomonas, and Staphylococcus were common. These isolated bacteria belonged to Proteobacteria, Firmicutes, Bacteroidetes, and Actinobacteria, and the fungi to Ascomycota, Basidiomycota, and Mucoromycota. Among the four shared bacteria isolated from six populations in this study, C. maltaromaticum were the most abundant culturable species both in the wild and laboratory-reared ghost moth larvae.

Taxonomic analysis revealed that the wild ghost moth gut bacterial community was dominated by Proteobacteria and Firmicutes $(>99 \%)$, while the laboratory-reared ghost moth gut bacterial community was mainly composed of Proteobacteria with significantly decreased abundance in Firmicutes, but increased abundance in Bacteroidetes and Actinobacteria (Figure 1). These four phyla were also the most common found in other lepidopteran species $[35,52-54]$. In this study, the fungal community of the ghost moth populations primarily consisted of Ascomycota and Basidiomycota, which was similar to other lepidopteran insects [53].

The composition of the ghost moth gut microbiota was unique. Each wild ghost moth population appeared to harbor a unique complex of microbes in their guts. Different host and growing environment (different altitude, diet and temperature) might be the factors that resulted in the changes among the wild ghost moth populations. For each population, the changed gut microbial communities were observed in the laboratory-reared population and the changes of each population due to artificial rearing appeared to be consistent within each of the samples. However, different gut microbial communities were found in the three laboratory-reared populations even though they were exposed to the same rearing conditions.

The wild populations had high abundance of Carnobacterium, particularly, the abundance of Carnobacterium in the wild SD samples reached up to $98.16 \%$ (Figure 1). Because Carnobacterium was detected in all samples, it might be a commensal inhabitant of the ghost moth. This inference was also supported by the presence of Carnobacterium in Thitarodes sp. unfertilized eggs and larvae $[21,39,55]$. Carnobacterium is a genus of Lactic Acid Bacteria (LAB), and C. maltaromaticum is frequently isolated from natural environment and foods [56]. It is able to grow at low temperatures, anaerobically and with increased $\mathrm{CO}_{2}$ concentrations [57]. Thus, it is reasonable to explain its overwhelming dominance in the gut of the wild ghost moth larvae on Tibet plateau. However, it is unclear whether the changed environment resulted in the significant decrease in the abundance of $C$. maltaromaticum in the laboratory-reared ghost moth. In addition, previous studies have shown that $C$. maltaromaticum is a probiotic bacterium extensively used in fish, meat, and dairy products, which can inhibit pathogenic and spoilage microorganisms [57]. It can also out-compete the pathogen and modulate the autochthonous midgut microbiota of Atlantic cod Gadus morhua [58]. More colonies of $C$. maltaromaticum were also detected in the gut of T. xiaojinensis larvae uninfected with $O$. sinensis than those injected with O. sinensis [21]. Therefore, whether C. maltaromaticum could be used as a probiotic bacterium to improve the growth of the ghost moth larvae needs further study.

When compared with the wild ghost moth larvae, the gut bacteria of laboratoryreared ghost moth larvae showed a significant increase in the abundance of Wolbachia and Rhizobium (Figure 6). Wolbachia is commonly found in about $80 \%$ of lepidopteran species [59]. Wolbachia is an intracellular bacterium, which is usually found in reproduction 
tissues, but it can also be found in different host tissues such as ovary, testis, fat body, midgut, Malpighian tubule, and leg [60]. It is considered as a parasite that could manipulate reproduction in Lepidoptera [61] and has also been found to inhabit insect cells and restrict viral infection in Drosophia melanogaster [62] and Aedes aegypti [63]. However, factors that resulted in significantly increased abundance of Wolbachia in the laboratory-reared ghost moth, especially in GG population, and the function of Wolbachia probably involved in the artificial rearing process are still unknown. In addition, some gut-associated bacterial genera also detected the increased abundance in the laboratory-reared ghost moth, for instance, Pseudomonas and Flavobacterium in SD population, and Serratia in XJ population. These genera are opportunistic pathogens in animal and/or humans and have also been found to facilitate other pathogens to infect host insects $[64,65]$. Therefore, the increased abundance in the laboratory-reared ghost moth should be carefully considered.

The infection mechanism of Thitarodes/Hepilus larvae by O. sinensis fungus is not well establish $[3,66]$. In the present study, it is suggested that $O$. sinensis may enter Thitarodes host by feeding as shared and high abundance of $O$. sinensis was present in the gut of the wild ghost moth populations from different geographic locations. However, significantly decreased abundance of $O$. sinensis and increased abundance of opportunistic pathogens Aspergillus and Candida were found in the laboratory-reared ghost moth populations. Interestingly, the most common pathogen I. farinosa was not detected in the gut of both the wild and laboratory-reared ghost moth. It seems that this fungus is an opportunistic pathogen for ghost moth larvae, as reported by Wu et al. [21]. In addition, I. farinosa could also lead the ghost moth larvae to death by being surface-treated with conidia suspension of I. farinosa (Figure S6).

\section{Conclusions}

The composition of the ghost moth gut microbiota is unique when compared with other insects. The results demonstrated that gut microbiota of the wild and laboratoryreared ghost moth populations greatly differed. The wild ghost moth larvae had a significantly higher abundance of Carnobacterium bacteria and Ophiocordyceps fungi in the gut compared with the laboratory-reared larvae. The gut of the laboratory-reared larvae was enriched by Wolbachia and other opportunistic pathogenic bacteria such as Serratia, Pseudomonas, and Flavobacterium, as well as opportunistic pathogenic fungi Aspergillus and Candida. These findings will help improve the effectiveness of the laboratory-reared Thitarodes hosts for the cultivation of the Chinese cordyceps, by selecting beneficial probiotics from the gut microbiota.

Supplementary Materials: The following are available online at https:/ /www.mdpi.com/article/10 .3390/insects12040327/s1: Figure S1: The geographical locations of the ghost moths sampled from the Tibetan alpine meadow in Google Earth 7.1; Figure S2: The phylogenetic tree of the ghost moth constructed by the mitochondrial cytochrome b gene using Neighbor-joining methods; Figure S3: Numbers of effective sequences and OTUs in each sample for bacteria (A) and fungi (B); Figure S4: Rank-abundance and rarefaction curves for all samples; Figure S5: PCR amplification on genomic DNA of the six groups with specific primers of Isaria farinose; Figure S6: Infection characteristics of the ghost moth caused by surface-treated with $10^{8}$ spores per $\mathrm{ml}$ of I. farinosa; Table S1: The percentages of the microbe species isolated from five different media at $13^{\circ} \mathrm{C}$ and $23^{\circ} \mathrm{C}$; Table S2: Sequencing summary for bacteria and fungi; Table S3: Comparison of the indices of alpha diversity between groups using Turkey's test; Table S4: Dissimilarity comparison of microbial community structures between group; Table S5: ITS analyses of isolates from Thitarodes sp. cadavers during the artificial cultivation.

Author Contributions: R.H. designed the research. G.L. and L.C. collected the samples. G.L., X.Z. and H.L. conducted the research. G.L. and Z.R. analyzed the data. G.L. and R.H. wrote the manuscript. All authors have read and agreed to the published version of the manuscript.

Funding: This research was funded by Natural Science Foundation of Guangdong Province (2018A030310489), Guangzhou Science and Technology Projects (201803010087), National Nature 
Science Foundation of China (31900368) and GDAS Special Project of Science and Technology Development (2018GDASCX-0107; 2019GDASYL-0105048).

Data Availability Statement: All the data analyzed has been submitted to the database with accession number described in this article.

Conflicts of Interest: The authors declare no conflict of interest.

\section{References}

1. Sung, G.H.; Hywel-Jones, N.L.; Sung, J.M.; Luangsa-ard, J.J.; Shrestha, B.; Spatafora, J.W. Phylogenetic classification of Cordyceps and the clavicipitaceous fungi. Stud. Mycol. 2007, 57, 5-59. [CrossRef] [PubMed]

2. Zhou, X.W.; Li, L.J.; Tian, E.W. Advances in research of the artificial cultivation of Ophiocordyceps sinensis in China. Crit. Rev. Biotechnol. 2014, 34, 233-243. [CrossRef]

3. Han, R.C.; Wu, H.; Tao, H.P.; Qiu, X.H.; Liu, G.Q.; Rao, Z.C.; Cao, L. Research on Chinese cordyceps during the past 70 years in China. Chin. J. Appl. Entomol. 2019, 56, 849-883.

4. Wiegmann, B.; Regier, J.; Mitter, C. Combined molecular and morphological evidence on the phylogeny of the lepidopteran lineages. Zool. Scr. 2002, 31, 67-81. [CrossRef]

5. Wang, D.P.; Li, H.G.; Li, Y.J.; Guo, S.C.; Yang, J.; Qi, D.L.; Jin, C.; Zhao, X.Q. Hypoxia-inducible factor $1 \alpha$ cDNA cloning and its mRNA and protein tissue specific expression in domestic yak (Bos grunniens) from Qinghai-Tibetan plateau. Biochem. Biophys. Res. Commun. 2006, 348, 310-319. [CrossRef]

6. Cui, X.; Gu, S.; Zhao, X.; Wu, J.; Kato, T.; Tang, Y. Diurnal and seasonal variations of UV radiation on the northern edge of the Qinghai-Tibetan Plateau. Agric. For. Meteorol. 2008, 148, 144-151. [CrossRef]

7. Wang, X.L.; Yao, Y.J. Host insect species of Ophiocordyceps sinensis: A review. ZooKeys 2011, 43-59.

8. Wang, H.S.; Zeng, H.; Xu, H.F. Study on change regulation of environment factors at Cordyceps sinensis growth area. Chin. Qinghai J. Anim. Vet. Sci. 2006, 36.

9. Nielsen, E.S.; Robinson, G.S.; Wagner, D.L. Ghost-moths of the world: A global inventory and bibliography of the Exoporia (Mnesarchaeoidea and Hepialoidea) (Lepidoptera). J. Nat. Hist. 2000, 34, 823-878. [CrossRef]

10. Tu, Y.Q.; Zhang, D.R.; Zeng, W.; Chen, S.J. Study on biological characteristic of Hepialus xiaojinensis in sichuan. Chin. J. Appl. Entomol. 2011, 48, 990-996.

11. Guo, X.; Liu, B.; Ma, S.B.; Gui, M.Y. The ecological environmental investigation and biological characteristic analysis of Cordyceps sinensis in Yunnan province. Edible Fungi China 2008, 27, 8-11.

12. Lu, Z.H.; Shi, P.; He, Y.C.; Zhang, D.L.; He, Z.Y.; Chen, S.J.; Tu, Y.Q.; Li, L.; Liu, F.; Zeng, W. Review on natural enemies and diseases in the artificial cultivation of Chinese caterpillar mushroom, Ophiocordyceps sinensis (Ascomycetes). Int. J. Med. Mushrooms 2015, 17, 693-700. [CrossRef]

13. Yang, R.H.; Wang, X.L.; Su, J.H.; Li, Y.; Jiang, S.P.; Gu, F.; Yao, Y.J. Bacterial diversity in native habitats of the medicinal fungus Ophiocordyceps sinensis on Tibetan plateau as determined using illumina sequencing data. FEMS Microbiol. Lett. $2015,362$. [CrossRef]

14. Zhu, W.; Zhang, H.; Li, X.; Meng, Q.; Shu, R.; Wang, M.; Zhou, G.; Wang, H.; Miao, L.; Zhang, J.; et al. Cold adaptation mechanisms in the ghost moth Hepialus xiaojinensis: Metabolic regulation and thermal compensation. J. Insect Physiol. 2016, 85, 76-85. [CrossRef]

15. Wu, W.; Sun, H.; Guo, J.; Jiang, F.; Liu, X.; Zhang, G. De novo transcriptome characterization of the ghost moth, Thitarodes pui, and elevation-based differences in the gene expression of its larvae. Gene 2015, 574, 95-105. [CrossRef]

16. Rao, Z.; Cao, L.; Qiu, X.; Han, R. Comparative transcriptome analysis reveals molecular strategies of ghost moth Thitarodes armoricanus in response to hypoxia and anoxia. J. Insect Physiol. 2019, 112, 23-34. [CrossRef] [PubMed]

17. Guo, L.X.; Xu, X.M.; Liang, F.R.; Yuan, J.P.; Peng, J.; Wu, C.F.; Wang, J.H. Morphological observations and fatty acid composition of indoor-cultivated Cordyceps sinensis at a high-altitude laboratory on Sejila mountain, Tibet. PLoS ONE 2015, 10, e0126095.

18. Liu, G.; Han, R.; Cao, L. Artificial cultivation of the Chinese cordyceps from injected ghost moth larvae. Environ. Entomol. 2019, 48, 1088-1094. [CrossRef]

19. Meng, Q.; Yu, H.Y.; Zhang, H.; Zhu, W.; Wang, M.L.; Zhang, J.H.; Zhou, G.L.; Li, X.; Qin, Q.L.; Hu, S.N.; et al. Transcriptomic insight into the immune defenses in the ghost moth, Hepialus xiaojinensis, during an Ophiocordyceps sinensis fungal infection. Insect Biochem. Mol. Biol. 2015, 64, 1-15. [CrossRef]

20. Rao, Z.C.; Cao, L.; Wu, H.; Qiu, X.H.; Liu, G.Q.; Han, R.C. Comparative transcriptome analysis of Thitarodes armoricanus in response to the entomopathogenic fungi Paecilomyces hepiali and Ophiocordyceps sinensis. Insects 2020, 11, 4. [CrossRef]

21. Wu, H.; Rao, Z.C.; Cao, L.; De Clercq, P.; Han, R.C. Infection of Ophiocordyceps sinensis fungus causes dramatic changes in the microbiota of its thitarodes host. Front. Microbiol. 2020, 11, 577268. [CrossRef] [PubMed]

22. Janson, E.M.; Stirema, J.O., 3rd; Singer, M.S.; Abbot, P. Phytophagous insect-microbe mutualisms and adaptive evolutionary diversification. Evolution 2008, 62, 997-1012. [CrossRef] [PubMed]

23. Sommer, F.; Bäckhed, F. The gut microbiota-masters of host development and physiology. Nat. Rev. Microbiol. 2013, 11, 227-238. [CrossRef] [PubMed] 
24. Coon, K.L.; Valzania, L.; McKinney, D.A.; Vogel, K.J.; Brown, M.R.; Strand, M.R. Bacteria-mediated hypoxia functions as a signal for mosquito development. Proc. Natl. Acad. Sci. USA 2017, 114, E5362-E5369. [CrossRef]

25. Hu, Y.; Sanders, J.G.; Lukasik, P.; D'Amelio, C.L.; Millar, J.S.; Vann, D.R.; Lan, Y.; Newton, J.A.; Schotanus, M.; Kronauer, D.J.C.; et al. Herbivorous turtle ants obtain essential nutrients from a conserved nitrogen-recycling gut microbiome. Nat. Commun. 2018, 9, 964. [CrossRef]

26. Thaiss, C.A.; Zmora, N.; Levy, M.; Elinav, E. The microbiome and innate immunity. Nature 2016, 535, 65-74. [CrossRef]

27. Martinson, V.G.; Danforth, B.N.; Minckley, R.L.; Rueppell, O.; Tingek, S.; Moran, N.A. A simple and distinctive microbiota associated with honey bees and bumble bees. Mol. Ecol. 2011, 20, 619-628. [CrossRef]

28. Ceja-Navarro, J.A.; Vega, F.E.; Karaoz, U.; Hao, Z.; Jenkins, S.; Lim, H.C.; Kosina, P.; Infante, F.; Northen, T.R.; Brodie, E.L. Gut microbiota mediate caffeine detoxification in the primary insect pest of coffee. Nat. Commun. 2015, 6, 7618. [CrossRef]

29. Francoeur, C.B.; Khadempour, L.; Moreira-Soto, R.D.; Gotting, K.; Book, A.J.; Pinto-Tomás, A.A.; Keefover-Ring, K.; Currie, C.R. Bacteria contribute to plant secondary compound degradation in a generalist herbivore system. mBio 2020, 11, e02146-20. [CrossRef]

30. Cheng, D.; Guo, Z.; Riegler, M.; Xi, Z.; Liang, G.; Xu, Y. Gut symbiont enhances insecticide resistance in a significant pest, the oriental fruit fly Bactrocera dorsalis (Hendel). Microbiome 2017, 5, 13. [CrossRef]

31. Engel, P.; Moran, N.A. The gut microbiota of insects-diversity in structure and function. FEMS Microbiol. Rev. 2013, 37, 699-735. [CrossRef]

32. Chouaia, B.; Goda, N.; Mazza, G.; Alali, S.; Florian, F.; Gionechetti, F.; Callegari, M.; Gonella, E.; Magoga, G.; Fusi, M.; et al. Developmental stages and gut microenvironments influence gut microbiota dynamics in the invasive beetle Popillia japonica Newman (Coleoptera: Scarabaeidae). Environ. Microbiol. 2019, 21, 4343-4359. [CrossRef]

33. Chen, S.; Zhang, D.; Augustinos, A.; Doudoumis, V.; Bel Mokhtar, N.; Maiga, H.; Tsiamis, G.; Bourtzis, K. Multiple factors determine the structure of bacterial communities associated with Aedes albopictus under artificial rearing conditions. Front. Microbiol. 2020, 11. [CrossRef]

34. Mikaelyan, A.; Dietrich, C.; Kohler, T.; Poulsen, M.; Sillam-Dusses, D.; Brune, A. Diet is the primary determinant of bacterial community structure in the guts of higher termites. Mol. Ecol. 2015, 24, 5284-5295. [CrossRef]

35. Martinez-Solis, M.; Collado, M.C.; Herrero, S. Influence of diet, sex, and viral infections on the gut microbiota composition of Spodoptera exigua caterpillars. Front. Microbiol. 2020, 11, 753. [CrossRef]

36. Li, W.J.; Dong, C.H.; Liu, X.Z.; Li, Q.P.; Xia, J.M.; Liang, F. Research advances in artificial cultivation of Chinese cordyceps. Mycosystema 2016, 35, 375-387.

37. Qin, Q.L.; Zhou, G.L.; Zhang, H.; Meng, Q.; Zhang, J.H.; Wang, H.T.; Miao, L.; Li, X. Obstacles and approaches in artificial cultivation of Chinese cordyceps. Mycology 2018, 9, 7-9. [CrossRef]

38. Liu, G.H.; Cao, L.; Qiu, X.H.; Han, R.C. Quorum sensing activity and hyphal growth by external stimuli in the entomopathogenic fungus Ophiocordyceps Sinensis. Insects 2020, 11, 205. [CrossRef] [PubMed]

39. Liu, L.; Wang, Z.K.; Yu, H.W.; Chen, S.J.; Yan, G.F.; Xia, Y.X. Analysis of the bacterial diversity in intestines of Hepialus gonggaensis larvae. Acta Mycol. Sin. 2008, 48, 616-622.

40. Yu, H.; Wang, Z.; Liu, L.; Xia, Y.; Yin, Y.; Yuan, Q.; Cao, Y.; Guoxiong, P. Analysis of fungal diversity in intestines of Hepialus gonggaensis larvae. Acta Microbiol. Sin. 2008, 48, 439-445.

41. Tao, Z.; Cao, L.; Zhang, Y.; Ye, Y.S.; Han, R.C. Laboratory rearing of Thitarodes armoricanus and Thitarodes jianchuanensis (Lepidoptera: Hepialidae), hosts of the Chinese medicinal fungus Ophiocordyceps sinensis (Hypocreales: Ophiocordycipitaceae). J. Econ. Eentomol. 2016, 109, 176-181. [CrossRef] [PubMed]

42. Zou, Z.W.; Liu, X.; Zhang, G.R. Revision of taxonomic system of the genus Hepialus (Lepidoptera, Hepialidae) currently adopted in China. J. Hunan Univ. Sci. Technol. 2010, 25, 114-120.

43. Cao, L.; Ye, Y.S.; Han, R.C. Fruiting body production of the medicinal Chinese caterpillar mushroom, Ophiocordyceps sinensis (Ascomycetes), in artificial medium. Int. J. Med. Mushrooms 2015, 17, 1107-1112. [CrossRef] [PubMed]

44. Andersen, K.; Kirkegaard, R.; Karst, S.; Albertsen, M. Ampvis2: An r package to analyse and visualise 16s rrna amplicon data. bioRxiv 2018. [CrossRef]

45. Segata, N.; Izard, J.; Waldron, L.; Gevers, D.; Miropolsky, L.; Garrett, W.S.; Huttenhower, C. Metagenomic biomarker discovery and explanation. Genome Biol. 2011, 12, R60. [CrossRef] [PubMed]

46. Zhang, G.R.; Yu, J.F.; Wu, G.G.; Liu, X. Factors influencing the occurrence of Ophiocordyceps sinensis. Acta Ecol. Sin. 2011, 31, 4117-4125.

47. Chu, H.F.; Wang, L.Y.; Han, H.X. Lepidoptera, Hepialidae and Epiplemidae in Fauna Sinica, Insecta; Beijing Science Press: Beijing, China, 2004; Volume 38, pp. 1-194.

48. Chao, A. Nonparametric estimation of the number of classes in a population. Scand. J. Stat. 1984, 11, 265-270.

49. Lemos, L.N.; Fulthorpe, R.R.; Triplett, E.W.; Roesch, L.F. Rethinking microbial diversity analysis in the high throughput sequencing era. J. Microbiol. Methods 2011, 86, 42-51. [CrossRef] [PubMed]

50. Liu, G.Q.; Han, R.C. Detection primer set, detection kit and detection method of Isaria farinosa in culture medium of Cordyceps sinensis. Chin. Pat. 2017, 201711340871.8.

51. Zhuo, F.P.; Chen, S.J.; Yin, Y.P.; Wang, Z.K.; Xia, Y.X. Analysis on the Hepialus gonggaensis's intestinal bacterial flora. J. Chongqing Univ. 2004, 27, 26-29. 
52. Liu, Y.; Shen, Z.; Yu, J.; Li, Z.; Liu, X.; Xu, H. Comparison of gut bacterial communities and their associations with host diets in four fruit borers. Pest Manag. Sci. 2020, 76, 1353-1362. [CrossRef]

53. Chen, B.; Du, K.; Sun, C.; Vimalanathan, A.; Liang, X.; Li, Y.; Wang, B.; Lu, X.; Li, L.; Shao, Y. Gut bacterial and fungal communities of the domesticated silkworm (Bombyx mori) and wild mulberry-feeding relatives. ISME J. 2018, 12, 2252-2262. [CrossRef]

54. Gong, Q.; Cao, L.J.; Sun, L.N.; Chen, J.C.; Gong, Y.J.; Pu, D.Q.; Huang, Q.; Hoffmann, A.A.; Wei, S.J. Similar gut bacterial microbiota in two fruit-feeding moth pests collected from different host species and locations. Insects 2020, 11, 840. [CrossRef] [PubMed]

55. Liang, Y.; Hong, Y.; Mai, Z.; Zhu, Q.; Guo, L. Internal and external microbial community of the Thitarodes moth, the host of Ophiocordyceps sinensis. Microorganisms 2019, 7, 517. [CrossRef] [PubMed]

56. Afzal, M.I.; Jacquet, T.; Delaunay, S.; Borges, F.; Millière, J.B.; Revol-Junelles, A.M.; Cailliez-Grimal, C. Carnobacterium maltaromaticum: Identification, isolation tools, ecology and technological aspects in dairy products. Food Microbiol. 2010, 27, 573-579. [CrossRef]

57. Leisner, J.J.; Groth, L.B.; Prévost, H.; Djamel, D.; Paw, D. Carnobacterium: Positive and negative effects in the environment and in foods. FEMS Microbiol. Rev. 2007, 31, 592-613. [CrossRef]

58. Martinsen, L.; Salma, W.; Myklebust, R.; Mayhew, T.; Ringoe, E. Carnobacterium maltaromaticum vs. Vibrio (Listonella) anguillarum in the midgut of Atlantic cod (Gadus morhua L.): An ex vivo study. Aquac. Res. 2011, 42, 1830-1839.

59. Ahmed, M.Z.; Araujo-Jnr, E.V.; Welch, J.J.; Kawahara, A.Y. Wolbachia in butterflies and moths: Geographic structure in infection frequency. Front. Zool. 2015, 12, 16. [CrossRef]

60. Narita, S.; Nomura, M.; Kageyama, D. Naturally occurring single and double infection with Wolbachia strains in the butterfly Eurema hecabe: Transmission efficiencies and population density dynamics of each Wolbachia strain. FEMS Microbiol. Ecol. 2007, 61, 235-245. [CrossRef] [PubMed]

61. Werren, J.H.; Baldo, L.; Clark, M.E. Wolbachia: Master manipulators of invertebrate biology. Nat. Rev. Microbiol. 2008, 6, 741-751. [CrossRef]

62. Teixeira, L.; Ferreira, A.; Ashburner, M. The bacterial symbiont Wolbachia induces resistance to RNA viral infections in Drosophila melanogaster. PLoS Biol. 2008, 6, 2753-2763. [CrossRef]

63. Ford, S.A.; Allen, S.L.; Ohm, J.R.; Sigle, L.T.; Sebastian, A.; Albert, I.; Chenoweth, S.F.; McGraw, E.A. Selection on Aedes aegypti alters Wolbachia-mediated dengue virus blocking and fitness. Nat. Microbiol. 2019, 4, 1832-1839. [CrossRef] [PubMed]

64. Wu, P.; Sun, P.; Nie, K.X.; Zhu, Y.B.; Shi, M.Y.; Xiao, C.G.; Liu, H.; Liu, Q.Y.; Zhao, T.Y.; Chen, X.G.; et al. A gut commensal bacterium promotes mosquito permissiveness to arboviruses. Cell Host Microbe 2019, 25, 110-112. [CrossRef] [PubMed]

65. Wei, G.; Lai, Y.L.; Wang, G.D.; Chen, H.; Li, F.; Wang, S.B. Insect pathogenic fungus interacts with the gut microbiota to accelerate mosquito mortality. Proc. Natl. Acad. Sci. USA 2017, 114, 5994-5999. [CrossRef]

66. Tu, Y.Q.; Zhang, D.L.; Zeng, W.; Chen, S.J.; Yin, D.H. Study on the infection of Cordyceps sinensis on the host larva Hepialus sp. Edible Fungi 2010, 3, 16-17. 